\title{
Imaging features of Burkitt lymphoma in pediatric patients
}

\author{
Betül Emine Derinkuyu \\ Öznur Boyunağa \\ Çiğdem Öztunalı \\ Funda Tekkeşin \\ Çağrı Damar \\ Ayşe Gül Alımlı \\ Arzu Okur
}

\begin{abstract}
Burkitt lymphoma is an aggressive and rapidly growing tumor that is curable and highly sensitive to chemotherapy. It can affect almost every tissue in the body, producing various clinical presentations and imaging appearances, according to the predilection of the different subtypes for certain sites. Awareness of its diagnostically specific imaging appearances plays an important role in rapid detection and treatment. In this pictorial review, we aimed to identify the most common imaging features of Burkitt lymphoma in pediatric patients.
\end{abstract}

From the Division of Pediatric Radiology (B.E.D. $\square$ betulemineyildiz@gmail.com, Ö.B., C.Ö., C.D., A.G.A), Department of Radiology, Gazi University School of Medicine, Ankara, Turkey; the Division of Pediatric Oncology (F.T., A.O.), Department of Pediatrics, Gazi University School of Medicine, Ankara, Turkey.

Received 20 May 2015; revision requested 15 June 2015; last revision received 7 July 2015; accepted 14 July 2015.

Published online 24 November 2015. DOI 10.5152/dir.2015.15211 urkitt lymphoma (BL) is an aggressive subtype of non-Hodgkin lymphoma (NHL) with a doubling time of 24-48 h. Even with its aggressive character, BL is curable and highly sensitive to chemotherapy. Thus, early diagnosis and treatment are critical before life-threatening complications, such as airway compromise or spinal cord compression, set in. Knowledge about the imaging appearances assists in rapid detection and treatment. In this pictorial review, we illustrated the most common imaging features of $\mathrm{BL}$.

In 1958, Denis Burkitt first described BL in Uganda, after encountering many children with rapidly growing jaw tumors. In 1964, some viral particles, termed Epstein-Barr virus (EBV), were identified in the tumor $(1,2)$. Since then, BL has been classified into three main clinical variants: endemic, sporadic, and immunodeficiency-associated. The endemic variant is linked to EBV infection and is observed particularly in Africa, where malaria is also endemic. The sporadic variant is the most common form in the rest of the world, where malaria in not endemic. The immunodeficiency-related type is observed after organ transplantation and in patients with human immunodeficiency virus infection or congenital immunodeficiency diseases, including Wiskott-Aldrich syndrome, ataxia telangiectasia, and X-linked lymphoproliferative disease (1-5).

The least common type of lymphoma in all age groups is generally BL with an incidence of $1 \%$. During childhood, it is the most frequent subtype of NHL with an incidence of $40 \%$. As $B L$ can involve various sites of the body, the clinical presentation and radiologic appearances differ; it may be localized or disseminated and can affect a wide variety of locations. In the endemic variant, head and neck involvement is more common; however abdominal involvement is more commonly observed in the sporadic form $(1,6)$.

\section{Head and neck involvement}

In the head and neck region, the predilection of $\mathrm{BL}$ for certain sites varies among different subtypes. In the endemic form, jaw and other facial bones are commonly affected by osteolytic lesions. On the other hand, Waldeyer ring (palatine and lingual tonsils, nasopharyngeal adenoids) and nodal involvement are commonly observed in the sporadic form (Fig. 1). The clinical presentation may differ as per the site involved. The clinical features of endemic BL involving the jaws include acute swelling in or around the jaws or orbit, severely mobile teeth, displaced teeth, generalized lymphadenopathy and anesthesia or paresthesia when adjacent nerves are involved; pain is not a prominent feature. On the other hand, in Waldeyer ring involvement, airway compression may cause symptoms such as dysphagia, dyspnea, wheezing, and airway obstruction, which may be potentially life threatening (Figs. 2, 3) (2).

Ultrasonography (US) may be the initial modality for enlarged lymph nodes in the neck. On the other hand, computed tomography (CT) or magnetic resonance imaging (MRI) not only identifies a possible Waldeyer ring involvement or bone destruction according to the sub- 
type, but also enables a precise definition of the radiologic extent of the disease for staging (Figs. 4, 5). CT is widely available and inexpensive with a short examination time and no need for sedation. CT is superior for the imaging of bone destruction; however, it has poor soft tissue contrast and potential hazard of radiation. In contrast, MRI provides very detailed diagnostic pictures of most important sites in the neck, with superior soft tissue contrast that could demonstrate tumor extension. MRI does not use ionizing radiation, and is therefore, suitable for use in children. In addition, MRI is superior in showing the signal characteristics of the bone marrow. For highly cellular BL, diffusion-weighted MRI is a powerful tool that demonstrates restricted diffusion (Fig. 4c). However, the disadvantages of MRI over CT are the cost, longer scan time and requirement for sedation in a child, which is not preferred if airway obstruction is present.

In the sporadic form of BL, Waldeyer ring is the most common site of extranodal disease. On MRI, soft tissue masses and enlarged pharyngeal or neck lymph nodes are commonly observed. The enlarged lymph nodes and soft tissue masses demonstrate homogenous enhancement without any central necrosis. Mass lesions do not have a tendency to cause skull base erosions in the sporadic form of BL but a tendency to fill in the adjacent spaces, such as the nasal cavity, nasopharynx, or oropharynx. On T2-weighted sequences, the signal of a mass is isointense to hypointense because of its high cellularity. For the same reason, diffusion-weighted images show restricted diffusion. After contrast administration, the mass and the enlarged lymph nodes

\section{Main points}

- The predilection of Burkitt lymphoma for certain sites differs among different subtypes.

In the endemic variant, head and neck involvement is more common; whereas abdominal involvement is commonly observed in the sporadic form.

- In the head and neck region, jaw and the other facial bones are commonly involved in the endemic form. Waldeyer ring (palatine and lingual tonsils, nasopharyngeal adenoid) and nodal involvement are commonly seen in the sporadic form.

- In the abdomen, ileocecal region (the Peyer's patches in particular) is the most common site of involvement, as characterized by an asymmetric bowel wall thickening and aneurysmal dilatation of the bowel lumen.
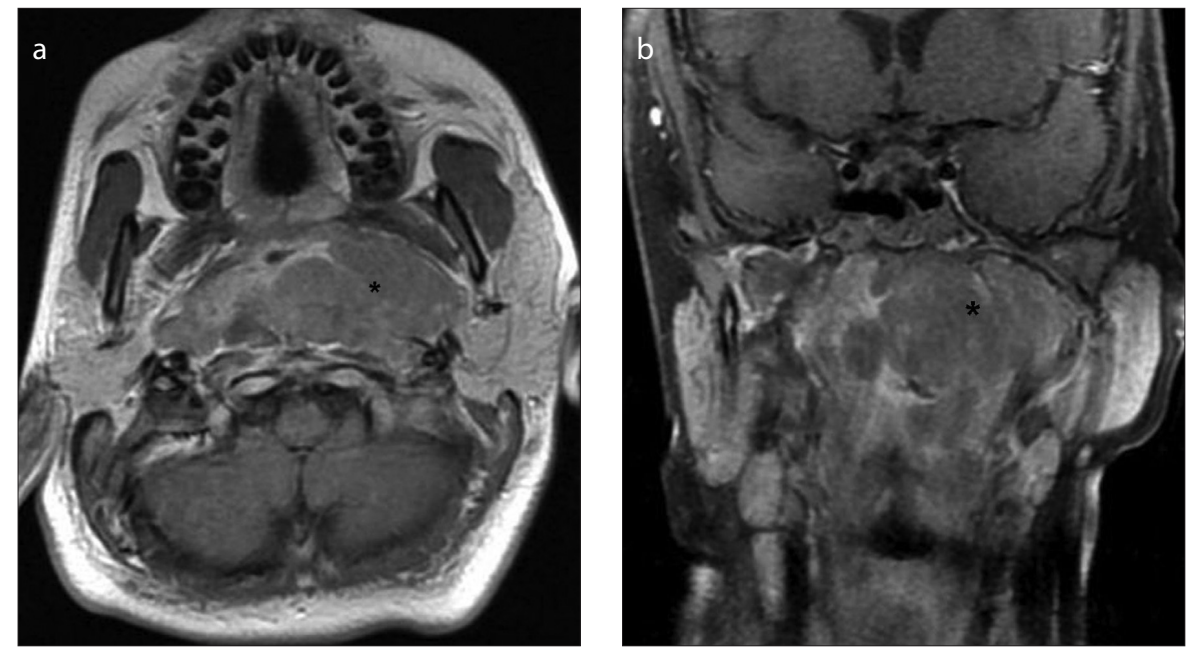

Figure 1. a, b. Nasopharyngeal MRI of a 15-year-old boy shows a left nasopharyngeal mass on the axial (a) and coronal (b) planes on T1-weighted postcontrast images. Note that the mass lesion is homogeneously enhanced without any necrosis (asterisk).
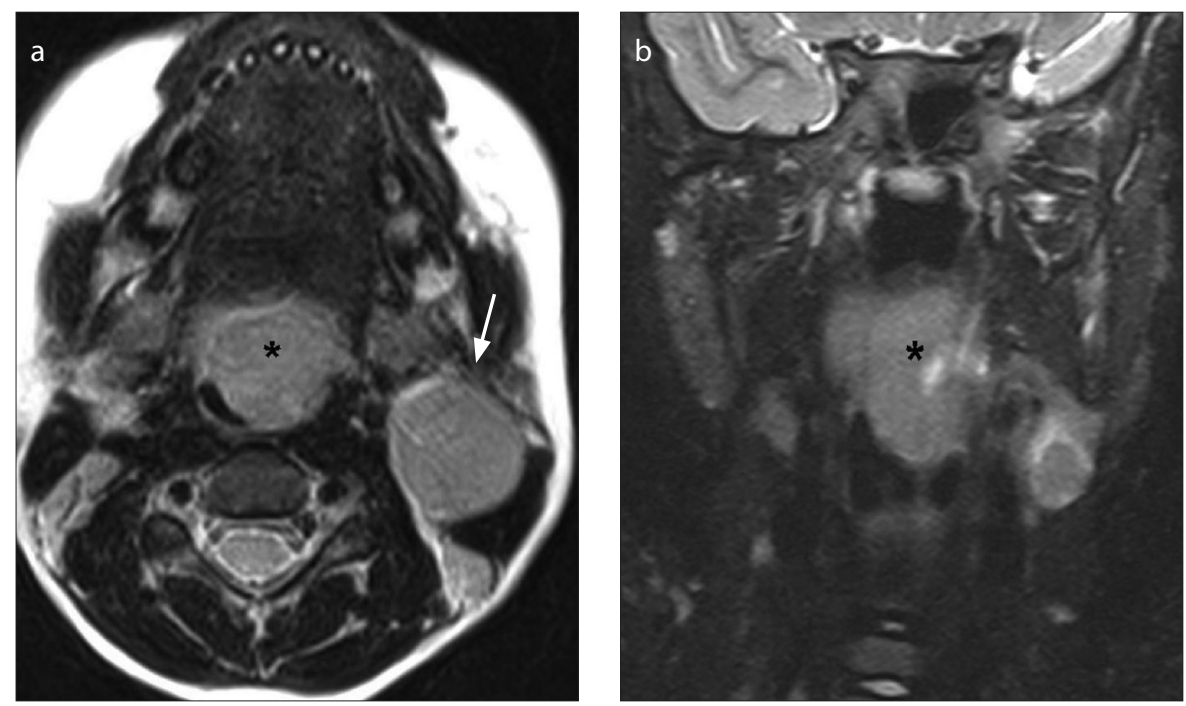

Figure 2. a, b. Nasopharyngeal MRI of a six-year-old girl shows a left tonsillar mass (asterisk) causing airway compromise, on axial plane T2-weighted (a) and coronal STIR (b) images. Note the enlarged lymph node (white arrow) in the left $2 \mathrm{~b}$ level (a).

enhance homogeneously. Because necrotic lymph nodes and bone destruction are prevalent in carcinomas of the head and neck, their absence supports the diagnosis of a sporadic form of BL in a child (6).

Unlike the sporadic form of $B L$, the endemic form commonly presents with bone destruction around the jaws and orbit. CT shows osteolytic lesions in the maxillofacial bones, particularly in the maxilla and mandible. When the mass grows, it enlarges into the adjacent areas, erodes the adjacent bones, and invades the adjacent spaces (Fig. 6) $(2,4,6)$.

Brain involvement is rare in $\mathrm{BL}$, particularly in the sporadic form. However, in immunodeficiency-related type of $B \mathrm{~L}$, involvement of the central nervous system and calvarial bone marrow may be observed (Fig. 7a-7c) $(2,4)$.

\section{Abdominal involvement}

The abdominal and pelvic region, including the gastrointestinal tract, mesentery, peritoneum, retroperitoneum, and genitourinary tract, is commonly affected by $\mathrm{BL}$, particularly by its sporadic form $(7,8)$. The ileocecal region, specifically the Peyer's patches, is the most common site involved. The clinical presentation may differ according to the site involved. Mainly, obstruction or compression may cause various symptoms, such as palpable mass, abdominal pain, nausea, vomiting, distension, constipation, or intestinal obstruction $(5,9)$. 

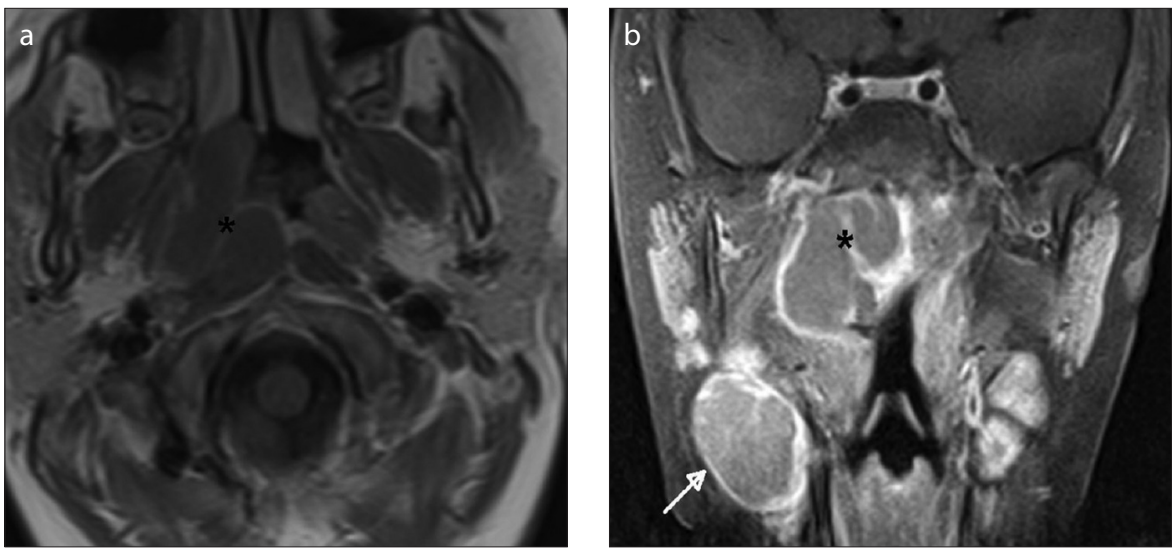

Figure 3. a, b. Nasopharyngeal MRI of a 10-year-old boy shows a right nasopharyngeal mass (asterisk) on the axial (a) and coronal (b) T1-weighted postcontrast images. Note that the mass lesion arises from nasopharyngeal adenoid tissue and is homogeneously enhancing, without any necrosis. Moreover, there is an enlarged lymph node (white arrow) that has similar signal characteristics with the mass on the right side of level $2 \mathrm{~b}(\mathbf{b})$.
The initial imaging modality for abdominal BL may be US for palpable masses, but $\mathrm{CT}$ is an important modality to evaluate the severity and spread of the disease for tumor staging. Abdominal CT should be performed with oral and intravenous contrast agents to evaluate the bowel walls, as well as solid organs, retroperitoneum, and the mesenteric region. Recently, positron emission tomography (PET)/CT has been used for functional and anatomic assessment during tumor staging and follow-up after treatment $(10,11)$.

Along the gastrointestinal tract, ileum, cecum, and appendix are common sites of involvement, because the terminal ileum has the highest concentration of lymphatic tissue; colonic and gastric involvement
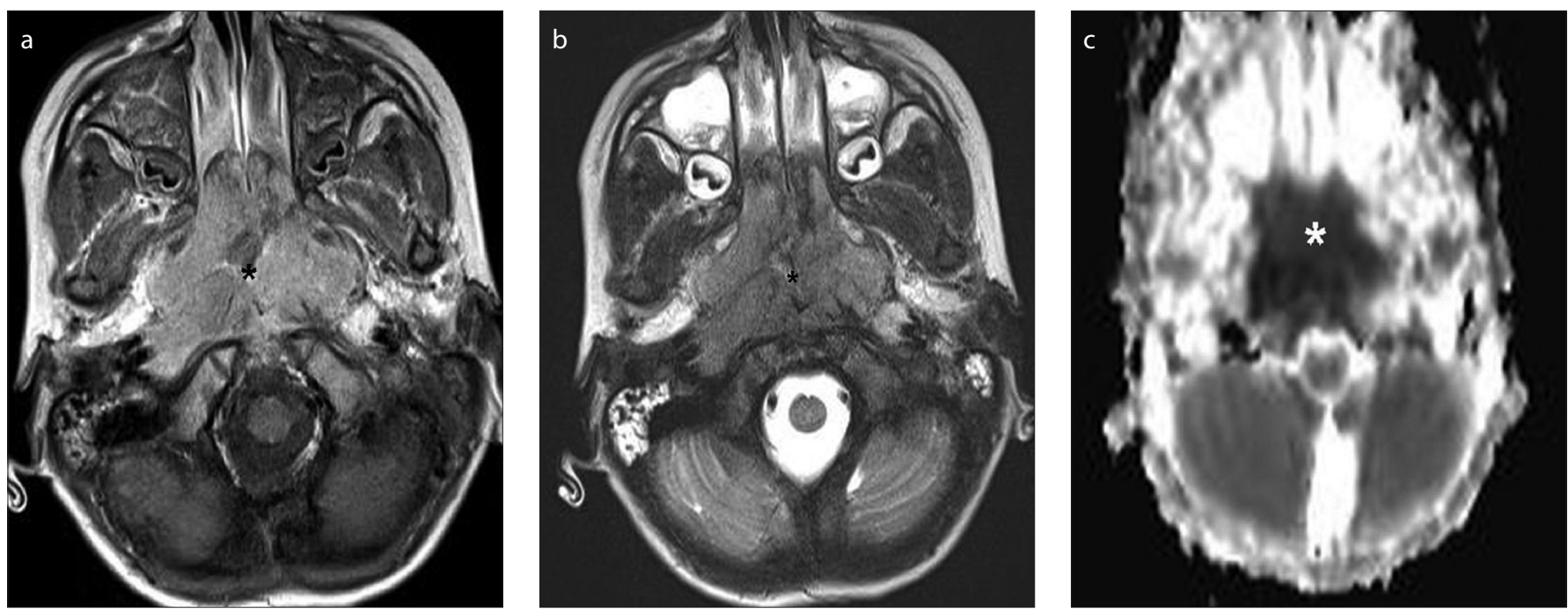

Figure 4. a-c. Nasopharyngeal MRI of a nine-year-old girl shows a midline nasopharyngeal mass (asterisk) filling the entire nasopharyngeal space on axial postcontrast T1-weighted (a) and T2-weighted images (b). Note that the mass lesion demonstrates restricted diffusion (asterisk) in the ADC map because of high tumor cellularity (c).
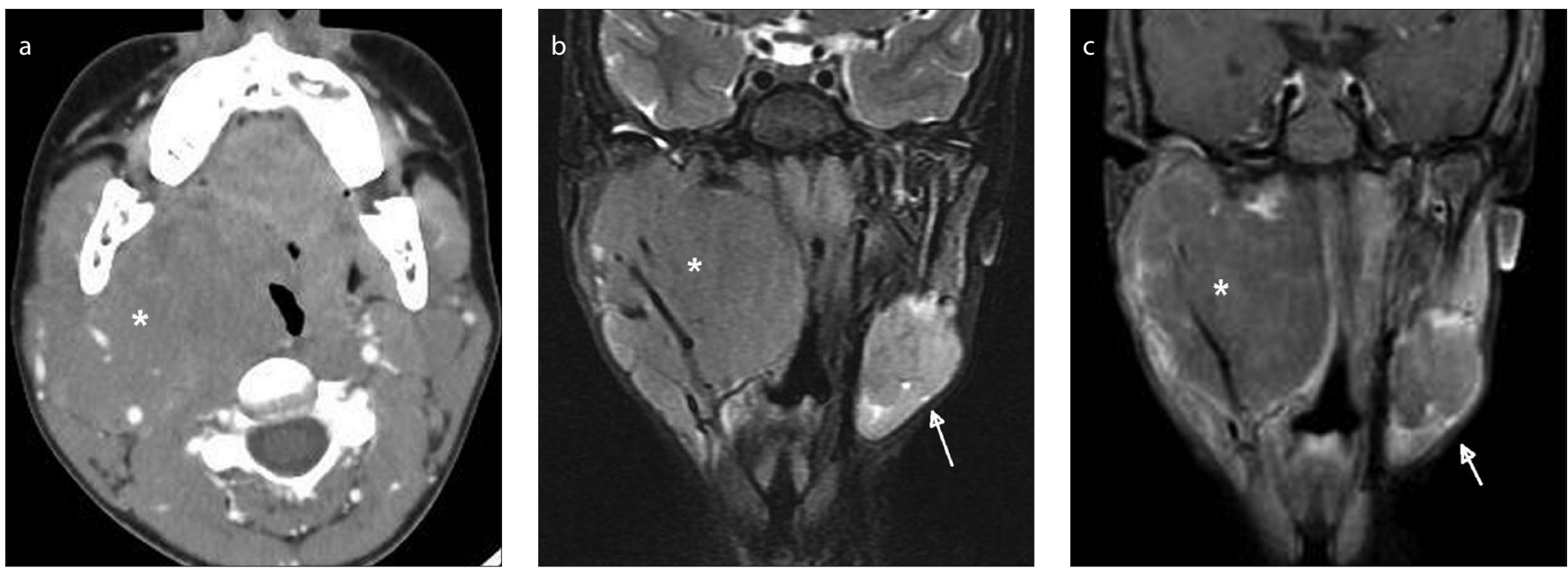

Figure 5. a-c. Contrast-enhanced maxillofacial CT (a) of an eight-year-old boy shows a large mass lesion (asterisk) located in the right infratemporal fossa causing displacement and narrowing of the airway. Fat-saturated T2-weighted (b) and postcontrast T1-weighted (c) coronal images demonstrate the border and extension of the mass lesion better than CT. Note the enlarged contralateral level 2a lymph node (white arrow) (c). 

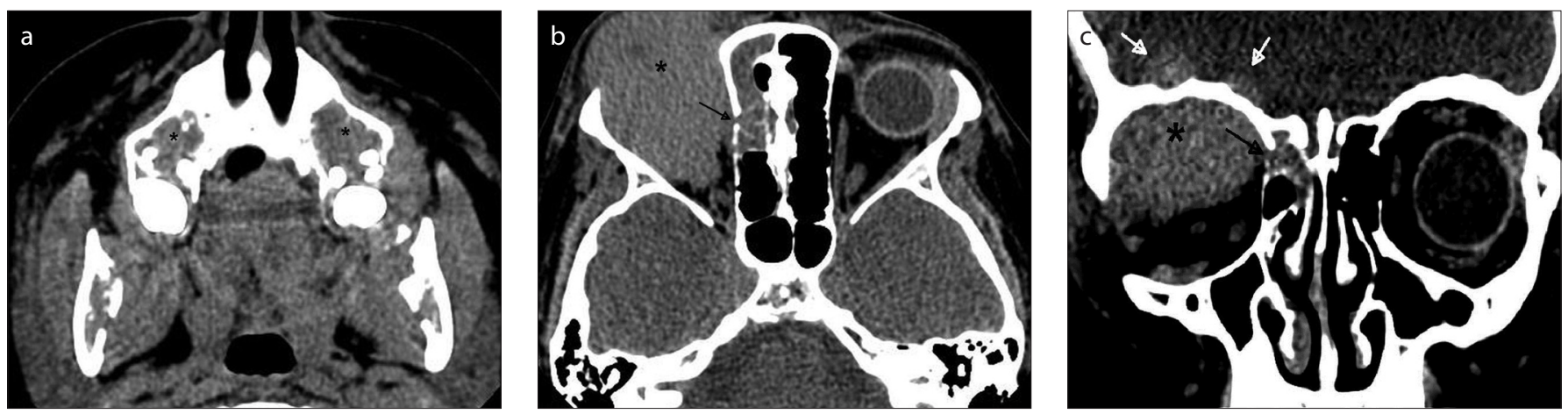

Figure 6. a-c. Maxillofacial CT (a) of a 10-year-old boy shows bilateral lytic and destructive lesions in the maxillary bone (asterisks). Unenhanced axial (b) and coronal (c) CT scans at the level of the orbit demonstrate a predominantly superior, extraconal mass lesion (asterisk) displacing the globe antero-inferiorly on the right. Note the extension of the mass lesion to the ethmoid sinus medially (black arrow) and to the anterior cranial fossa superiorly (white arrows).
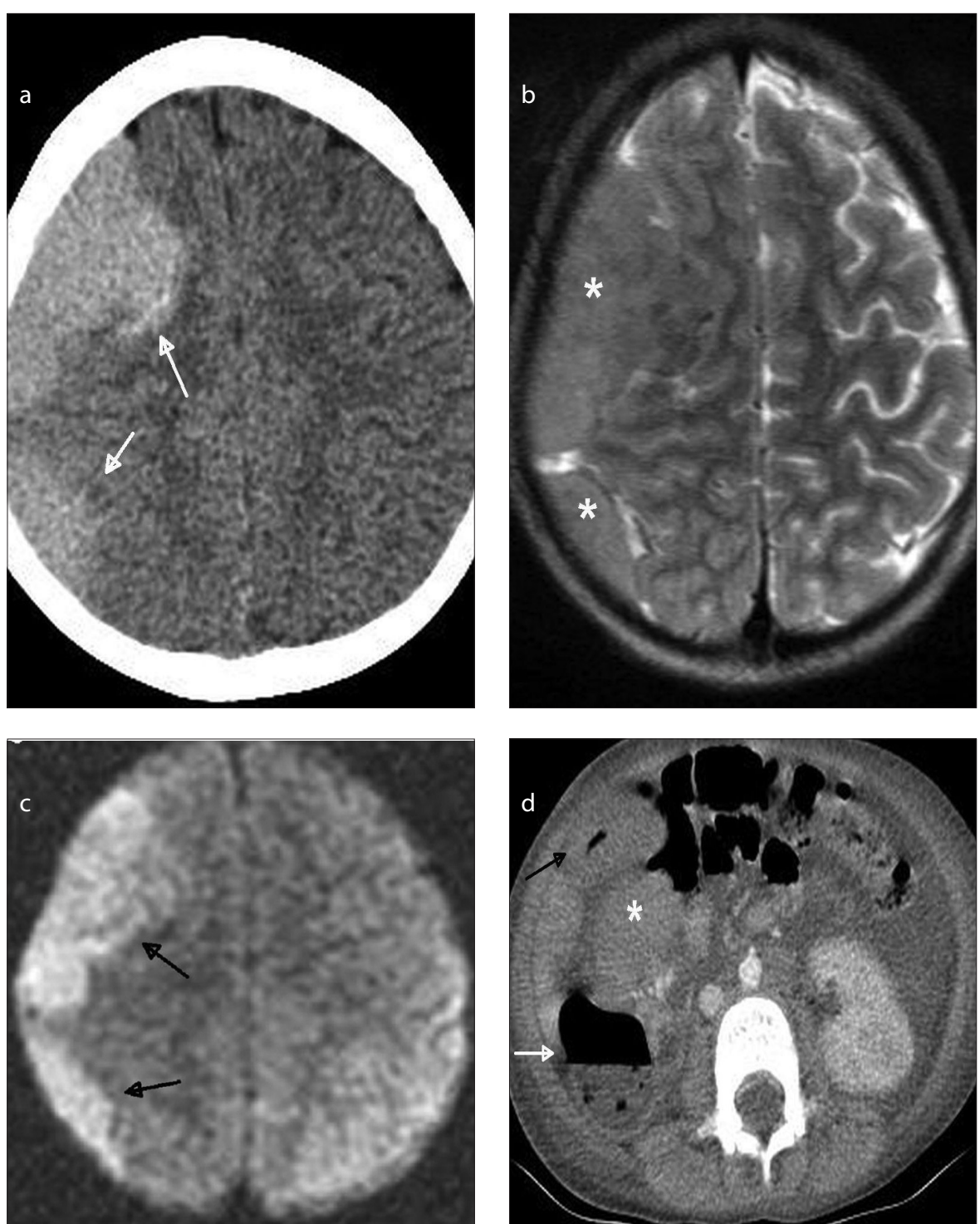

Figure 7. a-d. Unenhanced head CT (a) of a four-year-old boy, who had liver transplantation three years ago, demonstrates high-density dural mass lesions in the right frontoparietal region (white arrows), compatible with high-cellularity. Axial plane T2-weighted brain MRI (b) shows homogeneous, low-signal, lobulated dural mass lesions (asterisk) displacing the brain parenchyma. Note the restricted diffusion (black arrow) in the mass lesions corresponding to the higher tumor cellularity on diffusion-weighted image (c). Abdominal CT (d) of the same patient shows the high-density fluid collection as an exudate, the mass lesion (asterisk), the air-fluid level (white arrow) and the bowel wall thickening (black arrow). is quite rare (Fig. 8). Although BL does not cause bowel obstruction due to lack of fibrosis or desmoplasia unlike that in gastrointestinal adenocarcinomas, it may trigger intussusception and lead to bowel obstruction (Fig. 9) (12).

Imaging findings on US include asymmetric bowel wall thickening, solid hypoechoic mass lesion, ascites, or lymphadenopathies. If intussusception occurs, the pseudo-kidney sign on longitudinal plane and the target sign on transverse plane appear on sonogram. On CT, asymmetric bowel wall thickening and aneurysmal dilatation of the bowel lumen are observed more clearly. Aneurysmal dilatation is thought to be a consequence of infiltration of the myenteric plexus (Fig. 8c). In addition to these local findings, CT may detect ascites, peritoneal and mesenteric thickening, discrete or conglomerated masses, widespread lymphadenopathies, and tumor involvement of other solid organs in the abdomen, which leads to upstaging of the disease (Fig. 10). In BL, ascitic fluid can accumulate as an exudate, which has a slightly higher density on $\mathrm{CT}$, owing to its high protein content (Fig. 7d) $(5,9-11)$.

The typical mesenteric and retroperitoneal growth patterns in $\mathrm{BL}$ are infiltrative in nature. The involvement of mesenteric and retroperitoneal lymph nodes is observed as single or multiple abdominal or pelvis masses. Encasement of the major mesenteric vessels is a common feature (Fig. 10). Calcification and necrosis of the mesenteric and retroperitoneal masses may be observed in BL (5).

Although primary involvement of solid organs is rare, secondary involvement of the liver, spleen, or kidney is not uncommon. Radiologic findings include or- 

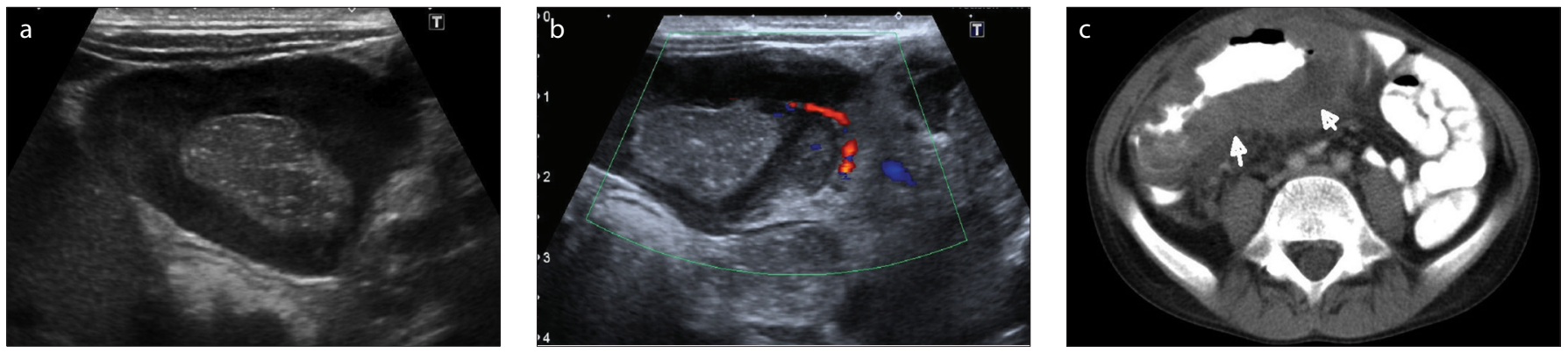

Figure 8. a-c. Abdominal US image (a) (9 MHz linear transducer; transverse plane) of a 12-year-old boy shows an asymmetric and hypoechoic bowel wall thickening at right lower quadrant. Color Doppler US (b) demonstrates vascularity of the thickened bowel wall. Abdominal CT with oral and intravenous contrast material (c) shows asymmetric bowel wall thickening and aneurysmal dilatation of the lumen at the level of the terminal ileum (white arrows).
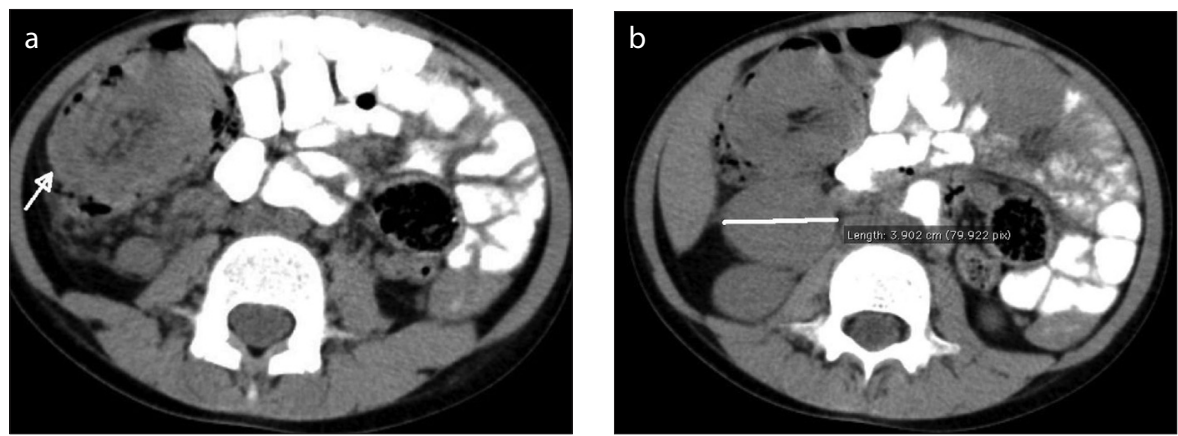

Figure 9. a, b. Abdominal CT (a, b) with oral and intravenous contrast in a seven-year-old girl with complaints of chronic anemia shows (a) chronic intussusception (white arrow) in the right lower quadrant. At the subhepatic level (b), note the enlarged lymph nodes located posteriorly, measuring almost $4 \mathrm{~cm}$ (white line).

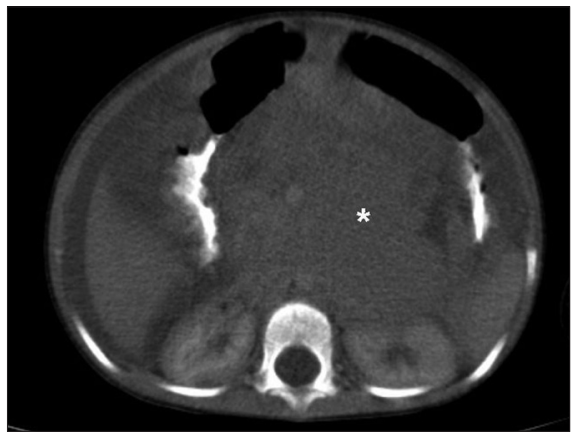

Figure 10. Abdominal CT with oral and intravenous contrast in a five-year-old boy shows a large mesenteric and retroperitoneal infiltrative mass (asterisk), consistent with conglomerated lymph nodes. Note the ascites.
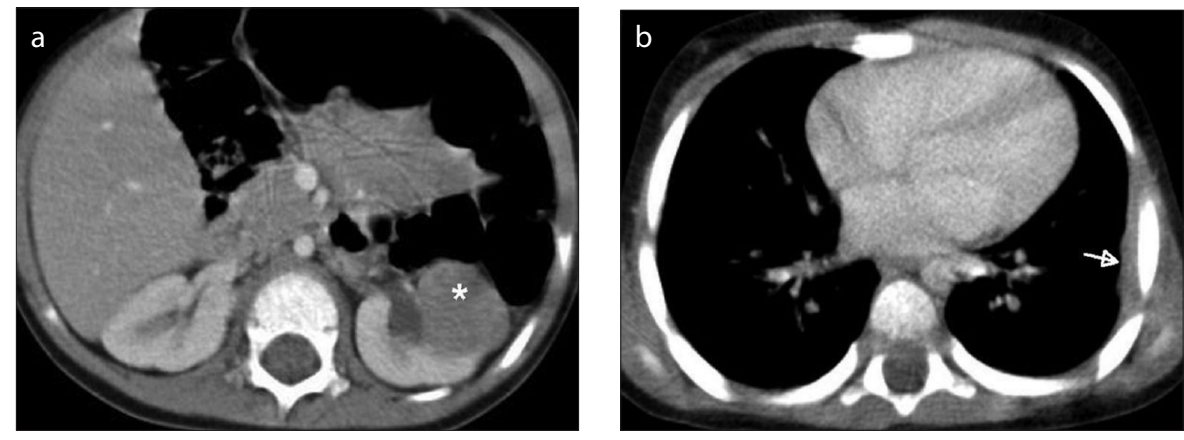

Figure 11. a, b. Abdominal CT (a) with intravenous contrast in a three-year-old boy with disseminated disease shows a hypodense mass lesion in the left kidney (asterisk). Chest CT (b) reveals asymmetric pleural thickening on the left lateral pleural surface (white arrow).

chymal hypoechogenicity-heterogeneity or focal hypoechoic mass in the testicular parenchyma. Ovarian involvement may be cystic or solid, or mixed, as a focal or large mass $(5,8)$.

Aside from abdominopelvic and headneck involvement, BL may seldom be located primarily in the pleura, breast, soft tissues, skin, thyroid, pancreas, lung, or spinal extradural area (Fig. 12). In disseminated disease, these tissues may be secondarily involved (Fig. 11b).

\section{Conclusion}

As an aggressive tumor, BL has to be rapidly detected among radiologic abnormalities and diagnosed, so that treatment can be initiated immediately. Moreover, diagnosis of $\mathrm{BL}$ requires a thorough radiologic investigation for accurate staging of the disseminated disease. Awareness of the most common imaging appearances and sites of involvement leads to rapid detection and correct diagnosis. ment, US is the primary imaging modality, which shows diffuse enlargement, paren- 


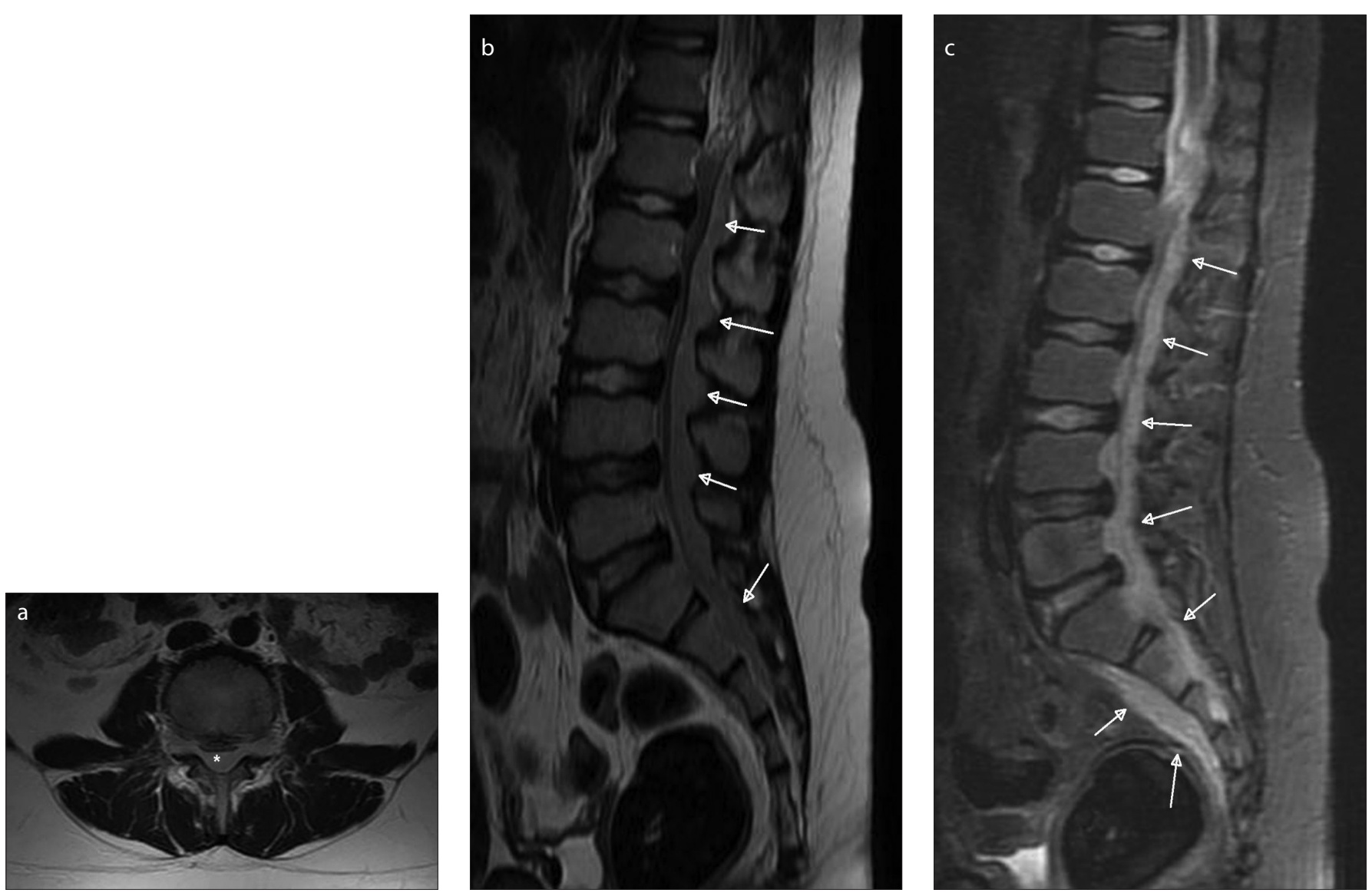

Figure 12. a-c. Axial plane T2-weighted lumbar MRI (a) of a nine-year-old boy with complaints of bladder and bowel dysfunction and lower extremity weakness, shows an epidural mass lesion (asterisk) filling the spinal canal, constricting the thecal sac totally and extending to the bilateral neural foramina. Sagittal T2-weighted image (b) and STIR sequence (c) demonstrate the mass lesion extending from the level of T12 to the level of S3 (white arrows). Note that the mass lesion is filling the entire spinal canal and spreads outward to the presacral area.

\section{Conflict of interest disclosure}

The authors declared no conflicts of interest.

\section{References}

1. Molyneux EM, Rochford R, Griffin B, et al. Burkitt's lymphoma. Lancet 2012; 379:12341244. [CrossRef]

2. Maeda E, Akahane M, Kiryu S, et al. Spectrum of Epstein-Barr virus-related diseases: a pictorial review. Jpn J Radiol 2009; 27:4-19. [CrossRef]

3. Vade A, Blane CE. Imaging of Burkitt lymphoma in pediatric patients. Pediatr Radiol 1985; 15:123-126. [CrossRef]

4. Tsui SHC, Wang MH, Lam WY. Burkitt's lymphoma presenting as mandibular swelling- report of a case and review of publications. Br J Oral Maxillofac Surg 2000; 38:8-11. [CrossRef]
5. Biko DM, Anupindi SA, Hernandez A, Kersun L, Bellah R. Childhood Burkitt Lymphoma: abdominal and pelvic imaging findings. AJR Am J Roentgenol 2009; 192:1304-1315. [CrossRef]

6. Thomas AG, Vaidhyanath R, Kirke R, Rajesh A. Extranodal lymphoma from head to toe: part 1 , the head and spine. AJR Am J Roentgenol 2011; 197:350-356. [CrossRef]

7. Kamona AA, El-Khatib MA, Swaidan MY, et al. Pediatric Burkitt's lymphoma: CT findings. Abdom Imaging 2007; 32:381-386. [CrossRef]

8. Lee WK, Lau EW, Duddalwar VA, Stanley AJ, Yo $\mathrm{HH}$. Abdominal manifestations of extranodal lymphoma: spectrum of imaging findings. AJR Am J Roentgenol 2008; 191:198-206. [CrossRef]

9. Dunnick NR, Reaman GH, Head GL, Shawker $\mathrm{TH}$, Ziegler JL. Radiographic manifestations of Burkitt's lymphoma in American patients. AJR Am J Roentgenol 1979; 132:1-6. [CrossRef]
10. Thomas AG, Vaidhyanath R, Kirke R, Rajesh A. Extranodal lymphoma from head to toe: part 2, the trunk and extremities. AJR Am J Roentgenol 2011; 197:357-364. [CrossRef]

11. Leite NP, Kased N, Hanna RF, et al. Cross-sectional imaging of extranodal involvement in abdominopelvic lymphoproliferative malignancies. Radiographics 2007; 27:1613-1634. [CrossRef]

12. Gupta H, Davidoff AM, Pui CH, Shochat SJ, Sandlung JT. Clinical implications and surgical management of intussusception in pediatric patients with Burkitt lymphoma. J Pediatr Surg 2007; 42: 998-1001. [CrossRef] 